\title{
Bloch Point Structure in a Magnetic Nanosphere
}

\author{
Oleksandr V. Pylypovskyi, ${ }^{1}$ Denis D. Sheka, ${ }^{1,2, \text { f }}$ and Yuri Gaididei ${ }^{2}$ \\ ${ }^{1}$ Taras Shevchenko National University of Kiev, 01601 Kiev, Ukraine \\ ${ }^{2}$ Institute for Theoretical Physics, 03143 Kiev, Ukraine
}

(Dated: September 25, 2018)

\begin{abstract}
A Bloch Point singularity can form a metastable state in a magnetic nanosphere. We classify possible types of Bloch points and derive analytically the shape of magnetization distribution of different Bloch points. We show that external gradient field can stabilize the Bloch point: the shape of the Bloch point becomes radial-dependent one. We compute the magnetization structure of the nanosphere, which is in a good agrement with performed spin-lattice simulations.
\end{abstract}

PACS numbers: 75.10.Hk, 75.40.Mg, 05.45.-a

\section{INTRODUCTION}

Topological singularities are widely recognized as key to understanding the behavior of wide variety condensed matter systems. Linear topological singularities such as dislocations, disclinations, and vortices, play a crucial role in low-dimensional phase transitions,$\frac{1}{1}$ crystalline ordering on curved surfaces ${ }^{2}$, rotating trapped BoseEinstein condesates ${ }^{3}$ etc. Recent advances in microstructuring technology have made it possible to fabricate various nanoparticles with well-prescribed geometry. Much recent research in this field has focused on the statics and dynamics of topological singularities in nanoscale confined systems: essentially inhomogeneous states can be realized in magnetic nanoparticles $4 \sqrt{6}$ and ferroelectric nanoparticles ${ }^{7}$. As a result of the competition between exchange and magnetic dipole-dipole interactions the ground state of magnetic disks with sizes larger than some tens of nanometers is a flux-closure vortex state.

Besides linear singularities there exist also so-called point singularities such as monopoles, Bloch points, boojums. For example, hedgehog (monopole) singularities play a crucial role in the behavior of matter near quantum phase transitions that are seen in a variety of experimentally relevant two-dimensional antiferromagnets, $\stackrel{8}{b}$ boojums are relevant in superfluid $\mathrm{He}-3,{ }^{9}$ Bloch points along with Bloch lines are principle in understanding of magnetic bubble dynamics ${ }^{4}[10$

The concept of point singularities was introduced in magnetism by Feldtkeller ${ }^{11}$, who considered different magnetization distributions inside the singularity and proposed first estimations of the Bloch point shape. Later Döring ${ }^{12}$ studies how magnetostatic energy governs the Bloch point structure by selecting the rotation angle inside the Bloch point. Bloch point singularities were directly observed in yttrium iron garnet crystals 13 During the last decade Bloch points were also studied by micromagnetic simulations in nanowires ${ }^{14}$ in bubble materials, $\frac{15}{15}$ in disks-shaped $\frac{16 \mid 17}{17}$ and astroid-shaped nanodots $\frac{18}{18}$. The ultrafast switching of the vortex core magnetization open doors to consider the vortex state nanoparticles as promising candidates for magnetic ele- ments of storage devices. There are different scenarios of the switching process: (i) The symmetric or so-called punch-through core reversal takes place under the action of DC magnetic field applied perpendicularly to the magnet plane $\frac{14|16| 19 \mid 20}{}$ This reversal process as a rule is mediated by creation of two Bloch points ${ }^{[16}$ However single Bloch point scenario was also mentioned in Thiaville et al.16. (ii) The switching under the action of different in-plane AC magnetic fields or by a spin polarized currents, $\underline{21} \underline{26}$ is accompanied by the temporary creation and annihilation of the vortex-antivortex pair. The latter is accompanied by Bloch point creation 17 .

The purpose of the current work is to study the magnetization structure of the Bloch point of the spherical nanosized particle. As opposed to bubble films, where the static Bloch point results from the transition between Bloch lines, $\frac{410}{10}$ and vortex nanodots, where the Bloch point dynamically appears during the vortex core switching process, $\frac{16121}{1}$ the Bloch point in the nanosphere is an example of "pure" singularity without surrounding. Such a singularity is in some respect the only stable singularity in ferromagnet. ${ }^{[16} \mathrm{We}$ consider different types of Bloch point and classify them in terms of vortex parameters. The conventional magnetization distribution in the Bloch point is generalized for the radial-dependent one. Such radial distribution becomes important for the Bloch point nanosphere under the action of nonhomogeneous magnetic field. We show that radial gradient field can stabilize the Bloch point and compute the magnetization structure, which is in a good agrement with performed spin-lattice simulations.

The paper is organized as follows. In Sec. I we describe the model and present the classification of different Bloch point types (Sec. IA). The energetic analysis and the Bloch structure is analyzed in Sec. IB. In order to stabilize the Bloch point inside the nanosphere, we consider the the influence of external gradient field on the magnetization structure. The Bloch point solution becomes radially dependent: we calculate the magnetization structure analytically in Sec. III In Sec. III we study the Bloch point structure numerically, in particular, the problem of stability. We discuss our results in Sec. [IV] In Appendix $\mathrm{A}$ we analyze the Bloch point structure under 
the influence of weak fields using the linearized equations.

\section{THE MODEL AND THE BLOCH POINT SOLUTIONS}

Let us consider the classical isotropic ferromagnetic sphere of the radius $R$. The continuum dynamics of the magnetization can be described in terms of the magnetization unit vector $\boldsymbol{m}=\boldsymbol{M} / M_{\mathrm{S}}=$ $(\sin \Theta \cos \Phi, \sin \Theta \sin \Phi, \cos \Theta)$, where $\Theta$ and $\Phi$ are, in general, functions of the coordinates and the time, and $M_{S}$ is the saturation magnetization. The total energy $E$ of such a sphere, normalized by $4 \pi M_{S}^{2} V$ with $V=\frac{4}{3} \pi R^{3}$ reads:

$$
\mathscr{E}=\mathscr{E}^{\mathrm{ex}}+\mathscr{E}^{\mathrm{f}}+\mathscr{E}^{\mathrm{ms}}
$$

The first term in 1a is dimensionless exchange energy:

$$
\mathscr{E}^{\mathrm{ex}}=\frac{3}{8 \pi} \varepsilon \int \mathrm{d} \boldsymbol{r}\left[(\boldsymbol{\nabla} \Theta)^{2}+\sin ^{2} \Theta(\boldsymbol{\nabla} \Phi)^{2}\right]
$$

with $\varepsilon=\ell^{2} / R^{2}$ being reduced exchange length, $\ell=$ $\sqrt{A / 4 \pi M_{S}^{2}}$ being the exchange length, $A$ being the exchange constant and $\boldsymbol{r}=(x, y, z) / R$ being the reduced radius-vector. The second term determines the interaction with external magnetic field $\boldsymbol{H}$ :

$$
\mathscr{E}^{\mathrm{f}}=-\frac{3}{4 \pi} \int \mathrm{d} \boldsymbol{r}(\boldsymbol{m} \cdot \boldsymbol{h}),
$$

where $\boldsymbol{h}=\boldsymbol{H} / 4 \pi M_{S}$ is a reduced external field. We will discuss the influence of external field later, see Sec. II. The last term determines the reduced magnetostatic energy:

$$
\mathscr{E}^{\mathrm{ms}}=-\frac{3}{8 \pi} \int \mathrm{d} \boldsymbol{r}\left(\boldsymbol{m} \cdot \boldsymbol{h}^{\mathrm{ms}}\right),
$$

where $\boldsymbol{h}^{\mathrm{ms}}=\boldsymbol{H}^{\mathrm{ms}} / 4 \pi M_{S}$ is a reduced magnetostatic field $\boldsymbol{H}^{\mathrm{ms}}$. Magnetostatic field $\boldsymbol{h}^{\mathrm{ms}}$ satisfies the Maxwell magnetostatic equations $4 / 5$

$$
\left\{\begin{array}{l}
\boldsymbol{\nabla} \times \boldsymbol{h}^{\mathrm{ms}}=0 \\
\boldsymbol{\nabla} \cdot \boldsymbol{h}^{\mathrm{ms}}=4 \pi \lambda
\end{array}\right.
$$

which can be solved using magnetostatic potential, $\boldsymbol{h}^{\mathrm{ms}}=-\boldsymbol{\nabla} \psi$. The source of the field $\boldsymbol{h}^{\mathrm{ms}}$ are magnetostatic charges: volume charges $\lambda \equiv-(\boldsymbol{\nabla} \cdot \boldsymbol{m}) / 4 \pi$ and surface ones $\sigma \equiv(\boldsymbol{m} \cdot \boldsymbol{n}) / 4 \pi$ with $\boldsymbol{n}$ being the external normal. The magnetostatic potential inside the sample reads:

$$
\begin{aligned}
& \psi(\boldsymbol{r})=\int_{V} \mathrm{~d} \boldsymbol{r}^{\prime} \frac{\lambda\left(\boldsymbol{r}^{\prime}\right)}{\left|\boldsymbol{r}-\boldsymbol{r}^{\prime}\right|}+\int_{S} \mathrm{~d} S^{\prime} \frac{\sigma\left(\boldsymbol{r}^{\prime}\right)}{\left|\boldsymbol{r}-\boldsymbol{r}^{\prime}\right|} \\
& \equiv \frac{1}{4 \pi} \int_{V} \mathrm{~d} \boldsymbol{r}^{\prime}\left(\boldsymbol{m}\left(\boldsymbol{r}^{\prime}\right) \cdot \nabla_{\boldsymbol{r}^{\prime}}\right) \frac{1}{\left|\boldsymbol{r}-\boldsymbol{r}^{\prime}\right|} .
\end{aligned}
$$

The equilibrium magnetization configuration is determined by minimization of the energy functional (1), which leads to the following set of equations:

$$
\varepsilon \nabla^{2} \boldsymbol{m}=\nabla \psi, \quad \nabla^{2} \psi=\nabla \cdot \boldsymbol{m} .
$$

\section{A. Classification of singularities}

Let us start the Bloch point as a particular solution of (4). In the exchange approach the simplest hedgehogtype Bloch point is characterized by the magnetization distribution of the form $\boldsymbol{m}=\boldsymbol{r} / r$ with a singularity at the origin. Using spherical frame of reference for the radius-vector $\boldsymbol{r}$ with the polar angle $\vartheta$ and azimuthal one $\varphi$, one can describe the magnetization angles of such a Bloch point as follows: $\Theta=\vartheta$ and $\Phi=\varphi$. The energy of the Bloch point in the exchange approach reads 12

$$
\mathscr{E}_{0}^{\mathrm{ex}}=3 \varepsilon, \quad E_{0}^{\mathrm{ex}}=4 \pi A R .
$$

This interaction is invariant with respect to the joint rotation of all magnetization vectors, which gives a possibility to consider family of solutions with different rotation angles. 1112

We consider the following singular magnetization distribution:

$$
\Theta(\vartheta)=p \vartheta+\pi(1-p) / 2, \Phi(\varphi)=q \varphi+\gamma, \quad p, q= \pm 1,
$$

which describes a three-parameter Bloch point. We refer to the parameter $q= \pm 1$ as to vorticity of the Bloch point and $p= \pm 1$ as to its polarity using the conventional symbols for magnetic vortices. The last parameter $\gamma$ describes the azimuthal rotational angle of the Bloch point! 1112

We refer to the micromagnetic singularity (6) as to $\mathrm{BP}_{q}^{p}$. For example, the hedgehog-type Bloch point is a vortex Bloch point with positive polarity $(p=1, q=1$, $\gamma=0)$. The schematic of magnetization distribution in different types of Bloch points is presented on Fig. 1. The analogy between Bloch point and vortices comes from the symmetric or punch-through vortex polarity switching process under the action of DC perpendicular magnetic field ${ }^{16}$ This reversal process as a rule is mediated by creation of two Bloch points ${ }^{16}$ For example, two singularities, $\mathrm{BP}_{1}^{1}$ and $\mathrm{BP}_{1}^{-1}$ describe intermediate state between two vortices with opposite polarities, see Fig. 1c. It is also instructive to mention that a single Bloch point can be imagined as a composite of two vortices with opposite polarities: such a singularity can appear in 3D Euclidean space during the vortex polarity switching process in antiferromagnets $8 \mid 27$. All four distributions for different signs of $p$ and $q$ can be observed during symmetrical Bloch points injection in polarity switching process of vortices 16 and antivortices 18 .

Topological properties of the Bloch point can be described by the topological (Pontryagin) index

$$
Q=\frac{1}{4 \pi} \int \sin \Theta(\boldsymbol{r}) \mathrm{d} \Theta(\boldsymbol{r}) \mathrm{d} \Phi(\boldsymbol{r})=p q .
$$




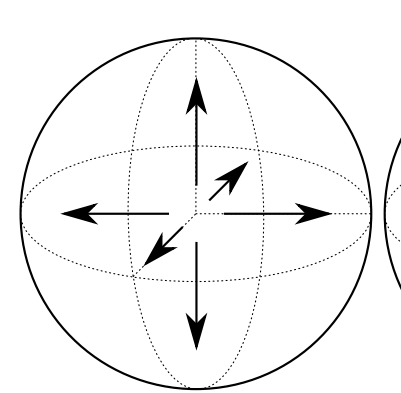

(a) $p=q=1$

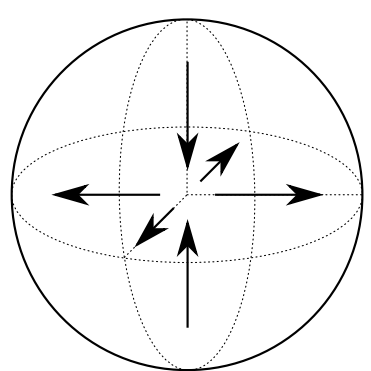

(b) $p=-1 q=1$

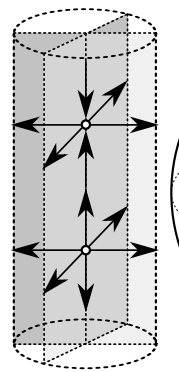

(c) Vortex switching

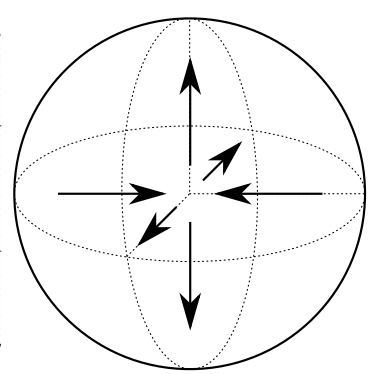

(d) $p=1 q=-1$

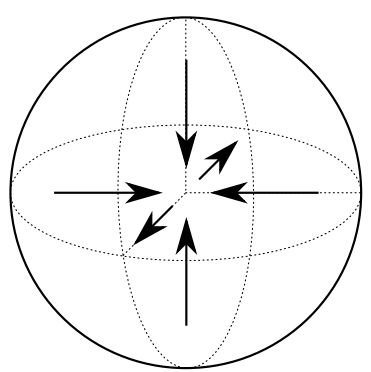

(e) $p=q=-1$

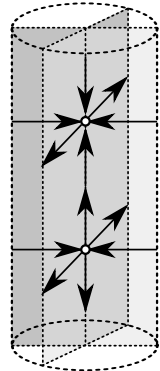

(f)

Antivortex switching

FIG. 1: Schematic of different types of Bloch points. Magnetization distribution in azimuthal vortex Bloch points in sphere, see Figs. 1a, 1b and both Bloch points in axial part of cylinder-shaped sample during the vortex polarity switching process, see Fig. 1c. The same is for azimuthal antivortex Bloch points, see Figs. 1d] Fig. 1e, and both singularities in axial part of astroid-shaped sample during the switching, see Fig. $1 \mathrm{ff}$.

Different Bloch point distributions with equal $Q$ are topologically equivalent: e.g., $\mathrm{BP}_{-1}^{-1}$ can be obtained from $\mathrm{BP}_{1}^{1}$ by simultaneous rotation of all magnetization vectors by $\pi$ in vertical plane, and $\mathrm{BP}_{-1}^{1}$ transforms to $\mathrm{BP}_{1}^{-1}$ by rotation by $\pi / 2$ in vertical plane. Note that similar topological notations were introduced by Malozemoff and Slonzewski $\frac{10}{10}$ for magnetic bubbles $\frac{28}{28}$

\section{B. Magnetization structure of Bloch points}

The most strong exchange interaction is invariant with respect to the rotation angle $\gamma$. Such degeneracy is removed under account of magnetostatic interaction. It is worth noting that the problem of stray field influence on the Bloch point energetics has a long story. Feldtkeller in his pioneer work ${ }^{111}$ used a so-called pole avoidance principle, see e.g. Ref. 29: the magnetostatic tries to avoid any sort of volume or surface charge. In this way he calculated the angle $\gamma$ from the condition that the total volume magnetostatic charge $\int \lambda(\boldsymbol{r}) \mathrm{d} \boldsymbol{r}=0$, where $\lambda(\boldsymbol{r})$ is the charge density. For the Bloch point given by Ansatz (6) it has a form $\lambda(\boldsymbol{r})=-\left[p \sin ^{2} \vartheta+\cos \gamma\left(\cos ^{2} \vartheta+1\right)\right] / 4 \pi r$ and leads to the rotation angle

$$
\gamma_{\mathrm{F}}=\arccos \left(-\frac{p}{2}\right)= \begin{cases}120^{\circ}, p=+1 \\ 60^{\circ}, \quad p=-1\end{cases}
$$

In it interesting to note that the same value $\gamma_{\mathrm{F}}$ also corresponds to absence of the total surface charge, $\int \sigma(\boldsymbol{r}) \mathrm{d} S=0$, where the surface charge density $\sigma(\boldsymbol{r})=$ $\left(p \cos ^{2} \vartheta+\cos \gamma \sin ^{2} \vartheta\right) / 4 \pi$.

Another approach was put forward by Döring $\frac{12}{12}$, who determined the equilibrium angle of $\gamma$ by minimizing the energy

$$
\mathscr{E}_{\mathrm{D}}^{\mathrm{ms}}=\frac{3}{8 \pi} \int_{V} \mathrm{~d} \boldsymbol{r}\left(h^{\mathrm{ms}}\right)^{2}
$$

and obtained

$$
\gamma_{\mathrm{D}}=\arccos \left(-\frac{11}{29}\right) \approx 112.3^{\circ} .
$$

However one has to emphasize that the equilibrium angle (10) minimizes only the inner part of the magnetostatic energy because the integration in $(9)$ is carried over the sample volume $V$ while the outer part of stray field is ignored. Note the similar approach was used in quite recent paper, 30 where a magnetization contraction was taken into account.

The aim of this section is to find the equilibrium rotation angle which minimizes the total magnetostatic energy. In order to derive the magnetostatic energy of Bloch points (6), we calculate first magnetostatic potential (3b) using an expansion of $1 /\left|\boldsymbol{r}-\boldsymbol{r}^{\prime}\right|$ over the spherical harmonics,

$$
\frac{1}{\left|\boldsymbol{r}-\boldsymbol{r}^{\prime}\right|}=\frac{1}{r_{>}} \sum_{l=0}^{\infty} \sum_{m=-l}^{l} \frac{4 \pi}{2 l+1}\left(\frac{r_{<}}{r_{>}}\right)^{l} Y_{l m}(\vartheta, \varphi) Y_{l m}^{\star}\left(\vartheta^{\prime}, \varphi^{\prime}\right)
$$

with $r_{<}=\min \left(r, r^{\prime}\right)$ and $r_{>}=\max \left(r, r^{\prime}\right)$ which results in

$$
\begin{aligned}
\psi_{q=1}^{p}(\boldsymbol{r}) & =p \pi r+\frac{\pi}{3}(9 r-8) \cos \gamma+\pi r(p-\cos \gamma) \cos ^{2} \vartheta, \\
\psi_{q=-1}^{p}(\boldsymbol{r}) & =p \pi r\left(1+\cos ^{2} \vartheta\right)+\pi r \cos (2 \varphi+\gamma) \sin ^{2} \vartheta .
\end{aligned}
$$

Simple calculations show that the magnetostatic energy of the antivortex Bloch point does not depend on $\gamma$ and $\mathscr{E}_{q=-1}=7 / 30 \approx 0.23$. In contrast to this, the vortex Bloch point energy depends on the rotation angle $\gamma$ and has the form

$$
\mathscr{E}_{q=1}^{\mathrm{ms}} p(\gamma)=\frac{1}{30}(7+4 p \cos \gamma+4 \cos 2 \gamma) .
$$

The equilibrium value of rotation angle $\gamma_{0}$ corresponds 


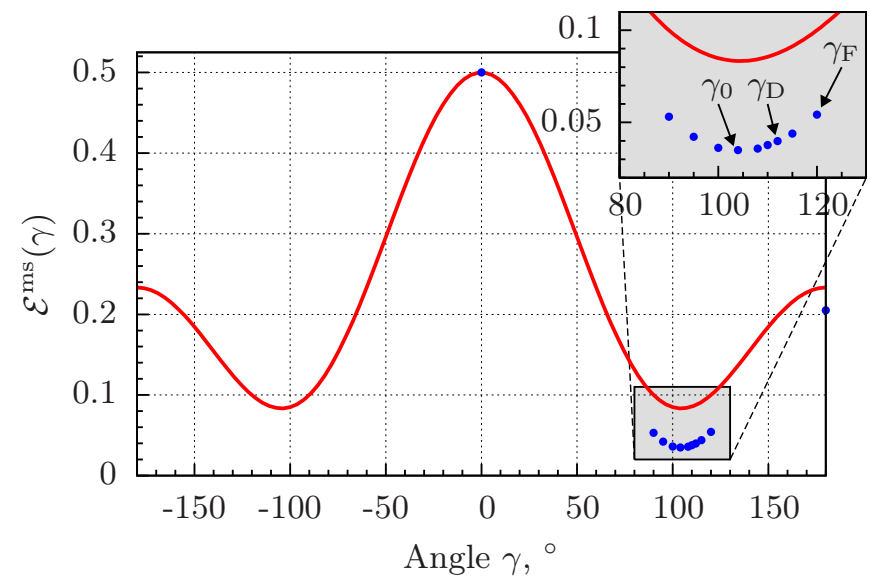

FIG. 2: (Color online.) The Bloch point energy vs rotation angle for $\mathrm{BP}_{1}^{1}$ : analytical result 112 ) (solid curve) and simulations (symbols). Simulations parameters: sphere diameter $2 R=35 a_{0}$, exchange

length $\ell=3.95 a_{0}$, damping parameter $\eta=0.5$.

to the minimum of the energy 12 . It gives

$$
\gamma_{0}=\arccos \left(-\frac{p}{4}\right) \approx\left\{\begin{array}{l}
105^{\circ}, p=+1 \\
76^{\circ}, p=-1
\end{array}\right.
$$

Let us compare Bloch point energies (12) for above mentioned approaches: the energy of Feldtkeller ${ }^{11}$ Bloch point $\mathscr{E}_{q=1}^{\mathrm{ms} p}\left(\gamma_{\mathrm{F}}\right)=0.1$, for Döring 12 Bloch point one has $\mathscr{E}^{\mathrm{ms}}\left(\gamma_{\mathrm{D}}\right) \approx 0.088$, the result by Elías and Verga is $\mathscr{E}_{1} \mathrm{~ms}\left(\gamma_{\mathrm{EV}}\right) \approx 0.089$. The minimal energy has a Bloch point with the rotation angle $\gamma_{0}$, see 13 :

$$
\mathscr{E}_{q=1}^{\mathrm{ms} p}\left(\gamma_{0}\right)=\frac{1}{12} \approx 0.083
$$

In order to verify our results we performed numerical spin-lattice simulations, see details in Sec. III. We compare analytical dependence $\mathscr{E}_{q=1}^{\mathrm{ms} p=1}(\gamma)$, see Eq. 12, with the discrete energy (24), extracted from simulations, see Fig. 2. Both dependencies are matched in maximum at $\gamma=0$. Comparison can be provided by calculating energy gain $\Delta \mathscr{E}(\gamma)=\mathscr{E}_{\max }-\mathscr{E} \mathrm{ms}(\gamma)$ for different rotation angles $\gamma$. According to simulation results the energy gain for mentioned above angles read:

$$
\Delta \mathscr{E}\left(\gamma_{\mathrm{F}}\right) \approx 0.446, \quad \Delta \mathscr{E}\left(\gamma_{\mathrm{D}}\right) \approx 0.460, \quad \Delta \mathscr{E}\left(\gamma_{0}\right) \approx 0.465
$$

The maximum energy gain takes place for $\gamma_{0}$, which corresponds to the energy minimum in a good agrement with our analytical result (13).

\section{THE BLOCH POINTS IN EXTERNAL FIELD}

The Bloch point does not form a ground state of a magnetic sphere. It corresponds to the saddle point (sphaleron) of the energy functional 31 . This brings up the question: How to stabilize the Bloch point? In this section we show that one way to achieve this goal is to apply a magnetic field which has the same symmetry as the hedgehog Bloch point with $\boldsymbol{m}=\boldsymbol{r} / r$, i. e. a radial symmetric magnetic gradient magnetic field in the form

$$
\boldsymbol{h}=b \boldsymbol{r} .
$$

Under the action of the space dependent magnetic field (15) the magnetization distribution also becomes space dependent. We take into account possible dependence by the following radial Bloch point Ansatz

$$
\Theta(\vartheta)=p \vartheta+\pi(1-p) / 2, \Phi(r, \varphi)=q \varphi+\gamma(r)
$$

with a radially dependent parameter $\gamma(r)$ in comparison with Eq. (6). The form of this Ansatz will justified by numerical simulations in Sec. III.

Inserting Eq. (16) into Eq. $\sqrt{1 b}$ for the exchange energy of such magnetization distribution we get

$$
\mathscr{E}^{\mathrm{ex}}=3 \varepsilon+\varepsilon \int_{0}^{1}\left(\frac{\mathrm{d} \gamma}{\mathrm{d} r}\right)^{2} r^{2} \mathrm{~d} r
$$

The magnetostatical potential of the Bloch point 16 reads

$$
\begin{aligned}
\psi_{q=1}^{p=1}(\boldsymbol{r}) & =-\frac{4 \pi}{3} \int_{r}^{1}\left[1+2 \cos \gamma\left(r^{\prime}\right)\right] \mathrm{d} r^{\prime}- \\
& -\frac{4 \pi}{3} \frac{3 \cos ^{2} \vartheta-1}{r^{3}} \int_{0}^{r} r^{\prime 3}\left[\cos \gamma\left(r^{\prime}\right)-1\right] \mathrm{d} r^{\prime} .
\end{aligned}
$$

Here and below we consider the case of $\mathrm{BP}_{1}^{1}$ only. The magnetostatic energy of such a Bloch point has the form

$$
\mathscr{E}^{\mathrm{ms}}=\frac{1}{10} \int_{0}^{1} r^{2}[7+4 \cos \gamma(r)+4 \cos 2 \gamma(r)] \mathrm{d} r .
$$

From Eq. 1c we obtain that the Bloch point interaction with magnetic field can be expressed as follows

$$
\mathscr{E}^{\mathrm{f}}=-2 b \int_{0}^{1} r^{3} \cos \gamma(r) \mathrm{d} r
$$

By minimizing the total energy, $\delta \mathscr{E} / \delta \gamma=0$, we obtain that the equilibrium distribution $\gamma(r)$ is a solution of the following nonlinear differential equation

$$
\varepsilon \frac{\mathrm{d}^{2} \gamma}{\mathrm{d} r^{2}}+\frac{2 \varepsilon}{r} \frac{\mathrm{d} \gamma}{\mathrm{d} r}+\frac{1}{5} \sin \gamma+\frac{2}{5} \sin 2 \gamma-b r \sin \gamma=0
$$

augmented by boundary conditions of the form

$$
\left.\frac{\mathrm{d} \gamma}{\mathrm{d} r}\right|_{r=0}=\left.\frac{\mathrm{d} \gamma}{\mathrm{d} r}\right|_{r=1}=0
$$




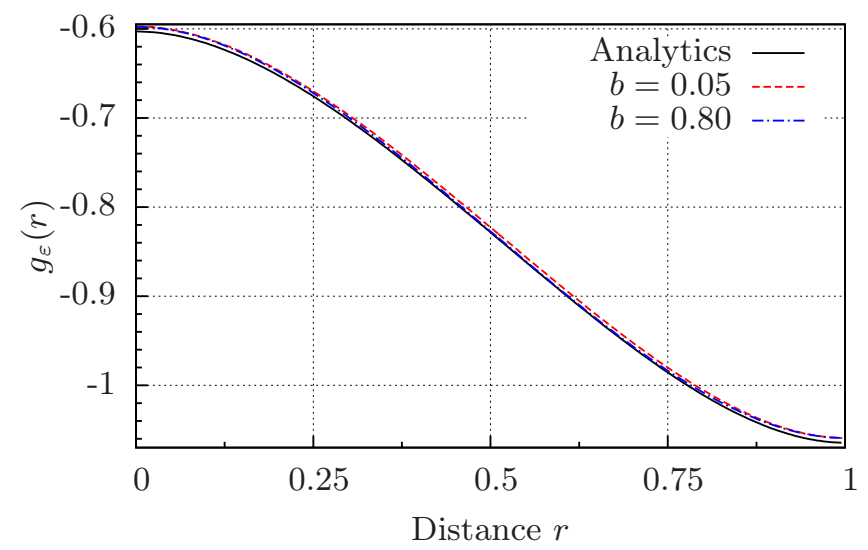

FIG. 3: (Color online.) Reduced rotation angle $g_{\varepsilon}(r)$, see 20 for different field intensities and $\varepsilon=0.05$ : analytical result A1) (solid curve) and numerical solution of (18) (dashed curves).

In the case of weak fields one can linearize Eq. $(18)$ in the vicinity of spatially uniform solution $\sqrt{13}$ and obtain that

$$
\gamma(r) \approx \gamma_{0}+b g_{\varepsilon}(r), \quad|b| \ll 1 .
$$

An explicit form of the function $g_{\varepsilon}(r)$ is calculated in Appendix A. The comparison with numerical solution of Eq. (18) shows a quite good agreement up to relatively strong fields $(b \lesssim 1)$, see Fig. 3 .

Another limiting case is realized in the case of strong magnetic fields when the Bloch point magnetization is parallel to the external field. In this case the rotation angle is $\gamma=0(\bmod \pi)$.

To describe the behavior of the Bloch point in a critical region $b \approx b_{c}$ where the spatially non-uniform distribution transforms to the spatially uniform one, we use a variational approach with a two-harmonics trial function $\gamma(\boldsymbol{r}) \approx \alpha_{0}+\alpha_{1} \cos \pi r$. Near the critical point $\alpha_{0}, \alpha_{1} \ll 1$. We expand the total energy in a Taylor series up to the fourth order with respect to $\alpha_{0}$ and to the second order with respect to $\alpha_{1}$. By excluding $\alpha_{1}$ and keeping terms not higher than $\alpha_{0}^{4}$, we get

$$
\mathscr{E}(\gamma) \approx \mathscr{E}_{0}+p_{2}(b, \varepsilon) \alpha_{0}^{2}+p_{4}(b, \varepsilon) \alpha_{0}^{4},
$$

The energy 21 as a function of $\alpha_{0}$ has a double-well shape $\left(p_{2}(b, \varepsilon)<0\right)$ for $b<b_{c}$ with the critical magnetic field $b_{c}$ given by

$$
b_{c}(\varepsilon) \approx 1.8-21.6 \varepsilon+\sqrt{0.4-20.2 \varepsilon+467 \varepsilon^{2}}
$$

In the critical region when $0<b_{c}(\varepsilon)-b \ll b_{c}(\varepsilon)$,

$$
\alpha_{0}(b) \approx a(\varepsilon) \sqrt{b_{c}(\varepsilon)-b}
$$

For $b>b_{c}, \quad p_{2}>0$ and the function Eq. 21 has a minimum for $\alpha_{0}=0$. It corresponds to $\gamma=0$. Numerical integration of Eq. 18 for $\varepsilon=0.05$ shows that the phase transition occurs when $b_{c} \approx 1.473$, see Fig. 4. It agrees well with the value $b_{c}(0.05) \approx 1.465$ obtained from Eq. 22, The critical behavior predicted by $(23)$ is also confirmed by our numerical simulations (see Fig. 4a).

\section{NUMERICAL STUDY OF THE BLOCH POINT STRUCTURE}

In order to check analytical results about Bloch point structure, we performed simulations using in-house developed spin-lattice simulator SLaSi ${ }^{32}$ that solves Landau-Lifshitz-Gilbert equation in terms of spins

$$
\frac{\mathrm{d} \boldsymbol{S}_{n}}{\mathrm{~d} t}=-\frac{1}{\hbar}\left[\boldsymbol{S}_{n} \times \frac{\partial \mathscr{H}}{\partial \boldsymbol{S}_{n}}\right]-\frac{\eta}{S}\left[\boldsymbol{S}_{n} \times \frac{\mathrm{d} \boldsymbol{S}_{n}}{\mathrm{~d} t}\right]
$$

where $\mathscr{H}$ is a lattice Hamiltonian of the classical ferromagnet:

$$
\begin{aligned}
& \mathscr{H}=-\frac{J}{2} \sum_{(n, \delta)} \boldsymbol{S}_{n} \cdot \boldsymbol{S}_{n+\delta}+2 \mu_{\mathrm{B}} \boldsymbol{H} \sum_{n} \boldsymbol{S}_{n} \\
& +2 \mu_{\mathrm{B}}^{2} \sum_{n \neq k}\left[\frac{\left(\boldsymbol{S}_{n} \cdot \boldsymbol{S}_{k}\right)}{r_{n k}{ }^{3}}-3 \frac{\left(\boldsymbol{S}_{n} \cdot \boldsymbol{r}_{n k}\right)\left(\boldsymbol{S}_{k} \cdot \boldsymbol{r}_{n k}\right)}{r_{n k}{ }^{5}}\right] .
\end{aligned}
$$

Here $\boldsymbol{S}_{n}$ is a classical spin vector with fixed length $S$ in units of action on the site $n$ of a three-dimensional cubic lattice with lattice constant $a_{0}, J$ is the exchange integral, $\mu_{\mathrm{B}}$ is Bohr magneton, $\boldsymbol{r}_{n k}$ is the radius-vector between $n$-th and $k$-th nodes, $\eta$ is a damping parameter, $\boldsymbol{H}$ is external magnetic field and $\delta$ runs over six nearest neighbors. Integration is performed by modified 4-5 order Runge-Kutta-Fehlberg method (RKF45) and free spins on the surface of the sample. 33

Numerically we checked the Bloch point structure, given by the radial-dependent Ansatz (16). by modeling spherically-shaped sample with diameter $2 R=35 a_{0}$ (such a sample consists of 24464 nodes with nonzero spin), and exchange length $\ell=3.95 a_{0}(\varepsilon=0.05)$. In order to stabilize the Bloch point we applied the gradient magnetic field with $b=1.0$. By modeling the overdamped dynamics we observed that the Bloch point structure quickly relaxes to the state similar to one, given by $(16)$ : The polar Bloch point angle $\Theta(\boldsymbol{r})$ does not deviate from $\vartheta$ within the accuracy 0.099 . The azimuthal angle is well also well-described by $(16)$ with the radial-dependent rotation angle $\gamma(r)$, see Fig. 5. Simulations were performed for crystallographic directions [111] $(\vartheta=\pi / 4)$ and $[110](\vartheta \approx \pi / 2$, the plane is shifted by $z=-0.5 a_{0}$ from the origin). One can see from Fig. 5 that numerical data are well confirmed by analytical curve $\gamma(r)$, calculated as numerical solution of 18 .

To validate our theory we performed also direct stability check. Numerically we check the stability of the Bloch point against the shift of its position. We start simulations with the Bloch point state using Ansatz-function (16), which is shifted along $\hat{z}$-axis by $\Delta z=-2 a_{0}$. We 


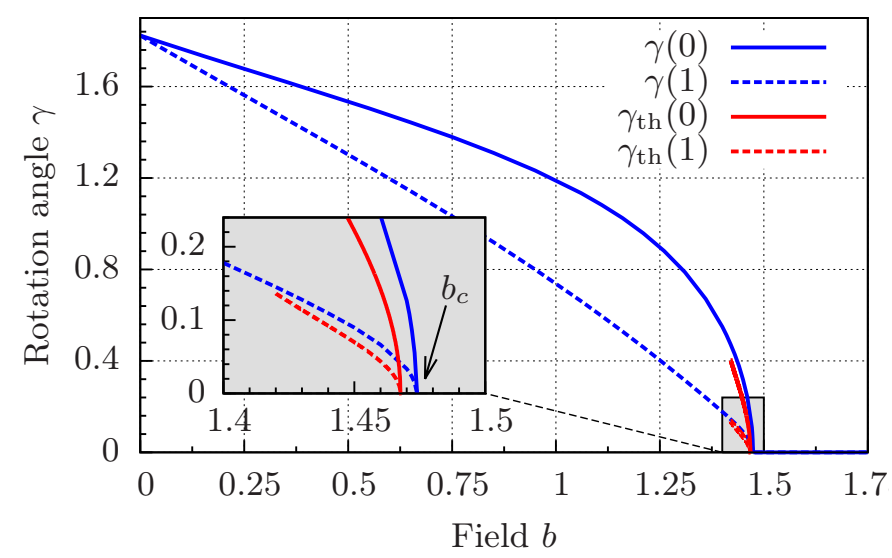

(a) Critical behaviour

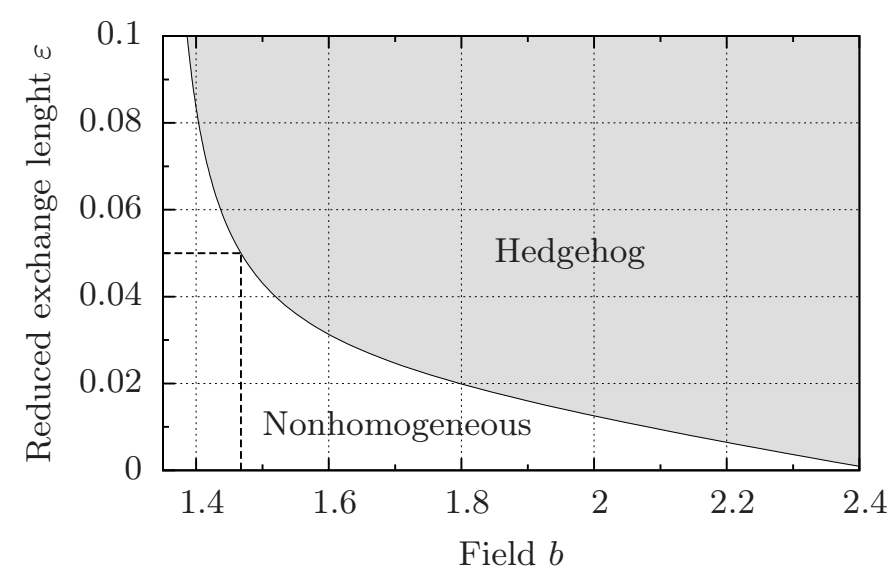

(b) Phase diagram

FIG. 4: (Color online.) Bloch point under the action of the gradient field. (a): Rotation angle vs field intensity $b$ near the critical field $b_{c} \approx 1.473$ from numerical solution of Eq. 18 (blue curves) and theoretical estimation by Eq. (23) (red curves) with $\varepsilon=0.05$. Solid lines correspond to the rotation angle $\gamma(0)$ and dashed line to $\gamma(1)$. (b): Phase diagram for solutions of Eq. (18). The upper (hedgehog) phase correspond to the solution $\gamma=0$, the lower (nonhomogeneous) one to the radial-dependent Bloch point with $\gamma(r)$. Dashed lines correspond to analytical result for the critical field $b_{c}=1.465$ for $\varepsilon=0.05$, see text.

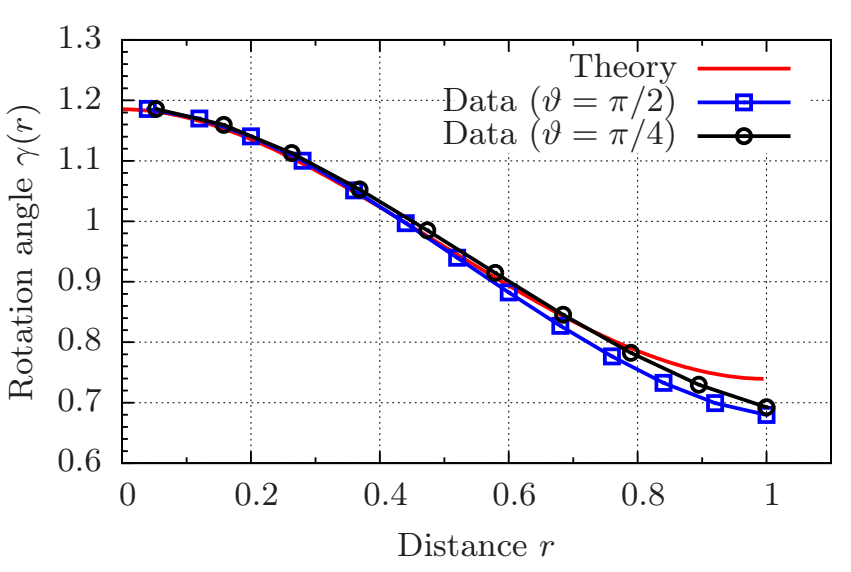

FIG. 5: (Color online.) Radial dependence of rotation angle $\gamma$ in spherical particle. Line: numerical integration of (18). Symbols: SLaSi simulations for crystallographic directions [110] and [111]. Parameters are the same as in Fig. 2 .

also apply $\gamma(r, t=0)=3^{\circ}$ in order to break the symmetry. For rapid relaxation we used in most of simulations the overdamped regime (the damping parameter $\eta=0.5)$. We checked the shift of the Bloch point by controlling the total spin projections: only for the Bloch point, situated at the sample origin, the total spin $S_{x}^{\text {tot }}=S_{y}^{\text {tot }}=S_{z}^{\text {tot }}=0$.

The temporal evolution of initially shifted Bloch point is presented in Fig. 6 for the Bloch point sample with $2 R=35 a_{0}(24456$ nodes $)$ in applied field with $b=1$, see also the supplementary vided $\underline{34}$. Originally the Bloch point was shifted down from the origin which corresponds to $S_{z}^{\text {tot }}>0$, see inset (a). During the evolution a number of magnons are generated, inset (b). After quick damping of oscillations, the micromagnetic singularity goes to the sample origin, see inset (c). The relaxation process consists of two parts: (i) The rotation angle $\gamma(r)$ changes its value from initial uniform one to the final nonhomogeneous state during a time $\tau_{\gamma} \approx 500 \omega_{0}^{-1}$. (ii) The relaxation of $S_{z}^{\text {tot }}$ component of total spin of the sample tooks approximately the same time. During all simulations time $\left|S_{x}^{\text {tot }}\right| \approx\left|S_{y}^{\text {tot }}\right| \lesssim 10^{-11}$.

\section{CONCLUSION}

To summarize, we study the magnetization structure of the Bloch point. In spite of the fact that the Bloch point as a simplest $3 \mathrm{D}$ topological singularity was studied during a long time, from the pioneer papers by Feldtkeller ${ }^{11}$ and Döring ${ }^{12}$, see also for review Refs. 4 and 10, the problem of the Bloch point structure still causes discussions $16130 / 35$ The point is that the most strong exchange interaction determines only the relative magnetization distribution accurate within the rotation angle $\gamma$. This rotation angle, which is determined by the magnetostatic interaction, is most questionable: its value is equal to $120^{\circ}$ according to Feldtkeller $\frac{11}{11}$, to $112.3^{\circ}$ following Döring ${ }^{12}$ and $113^{\circ}$ following Elías and Verga ${ }^{30}$. We analyze the origin of all these results and calculated the equilibrium value, about $105^{\circ}$, see $(12)$, which minimizes the total magnetostatic energy, not only the part 
Dynamics of $S_{z}^{\text {tot }}$ and plane $z=-0.5 a_{0}$

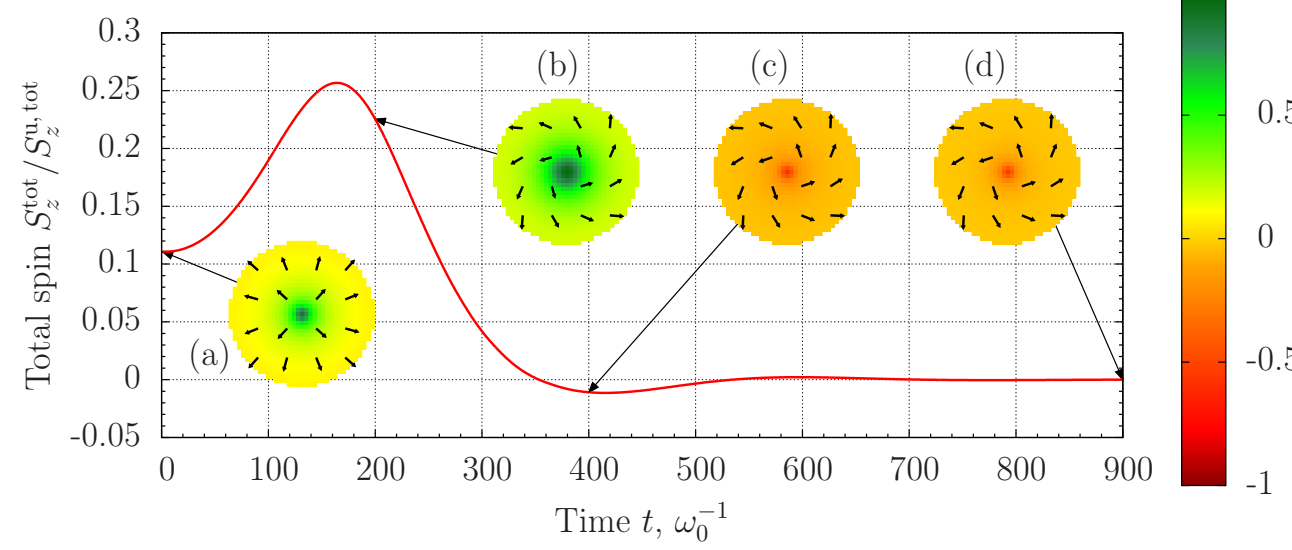

Plane $y=-0.5 a_{0}$
(a) $t=\omega_{0}^{-1}$
(b) $t=200 \omega_{0}^{-1}$
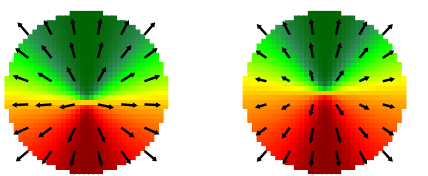

(c) $t=400 \omega_{0}^{-1}$ (d) $t=900 \omega_{0}^{-1}$
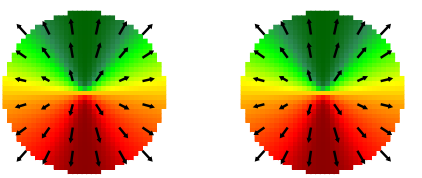

FIG. 6: (Color online.) Dynamics of total spin along $z$-axis of the sample. The Bloch point is initially shifted by $\Delta z=-2 a_{0}$ from center of the sample. Insets show magnetization distribution in $z=-0.5 a_{0}$ and $y=-0.5 a_{0}$ planes in different times. Color bar indicates $S_{z, n}$ for different lattice nodes. Applied field amplitude $b=1$, other parameters are the same as in Fig. 2

of it.

The next problem appears in modeling of the Bloch point. As is was discussed by Thiaville et al. 16 , the modeling of singularity is mesh-dependent. In particular, a mesh-friction effect and a strong mesh dependence of the switching field during the Bloch-point-mediated vortex switching process was detected using OOMMF micromagnetic simulations. $\frac{16}{16}$ The reason is that micromagnetic simulators consider the numerically discretized LandauLifstitz equation, which are valid in continuum theory. Since the Bloch point appears as a singularity of continuum theory, it is always located between mesh points, and causes the mesh-dependent effects and therefore may be insufficient for describing near-field Bloch point distribution. In contrast to this, spin-lattice simulations are free from these shortage. From the beginning we consider discrete spins, located on the cubic lattice, and their dynamics is governed by the discrete versions of LandauLifshitz equations. The lattice Hamiltonian allows us to calculate the discrete energy of the Bloch point similar to the atomiclike calculations by Reinhardt 36 .

Using in-house developed spin-lattice SLaSi ${ }^{32}$ simulator we modeled the Bloch point state nanosphere and checked our analytical predictions about Bloch point structure. We stabilized the singularity inside the spherical particle by applied gradient magnetic field. The field causes the new type of Bloch point with radial-dependent rotation angle $\gamma(r)$.

\section{ACKNOWLEDGMENTS}

Authors acknowledge computing time on the highperformance computing cluster of National Taras Shevchenko University of Kyiv ${ }^{37}$ and SKIT-3 Computing Cluster of Glushkov Institute of Cybernetic of NAS of Ukraine ${ }^{38}$. This work was supported by the Grant of the President of Ukraine No. F35/538-2011. We thank V. Kravchuk for helpful discussions.

\section{Appendix A: Bloch point structure in a weak field}

We consider here the magnetization structure of a Bloch point under the action of weak magnetic field. One has to linearize Eq. (18) on the background of the unperturbed rotation angle $\gamma_{0}$, see $(20)$, which can be presented as follows:

$$
\gamma(r) \approx \gamma_{0}+b g_{\varepsilon}(r), \quad g_{\varepsilon}(r)=\frac{2 \sqrt{5 \varepsilon}}{3} f(\lambda r), \quad \lambda=\frac{1}{2} \sqrt{\frac{3}{\varepsilon}} .
$$

Here the function $f(\xi)$ satisfies the linearized version of Eq. 18):

$$
\frac{\mathrm{d}^{2} f}{\mathrm{~d} \xi^{2}}+\frac{2}{\xi} \frac{\mathrm{d} f}{\mathrm{~d} \xi}-f=\xi
$$

which can be easily integrated:

$$
\begin{aligned}
f(\xi) & =C_{\lambda} \frac{\sinh \xi}{\xi}+2 \frac{\cosh \xi-1}{\xi}-\xi, \\
C_{\lambda} & =\frac{\lambda^{2}-2 \lambda \sinh \lambda+2 \cosh \lambda-2}{\lambda \cosh \lambda-\sinh \lambda} .
\end{aligned}
$$

The graphics of the $g_{\varepsilon}(r)$ for $\varepsilon=0.05$ is presented in Fig. 3 together with numerical solution of Eq. (18) by shooting method. In spite of limitation of our analysis by the case of weak field, $|b| \ll 1$, the function $g_{\varepsilon}(r)$ provides a good approximation for the solution of nonlinear Eq. (18) up to to very strong fields $b \leq 1$ with a relative error $\left|\left[\gamma(r)_{\text {num }}-\gamma(r)_{\text {theor }}\right] / \gamma(r)_{\text {num }}\right| \leq 0.04$. 


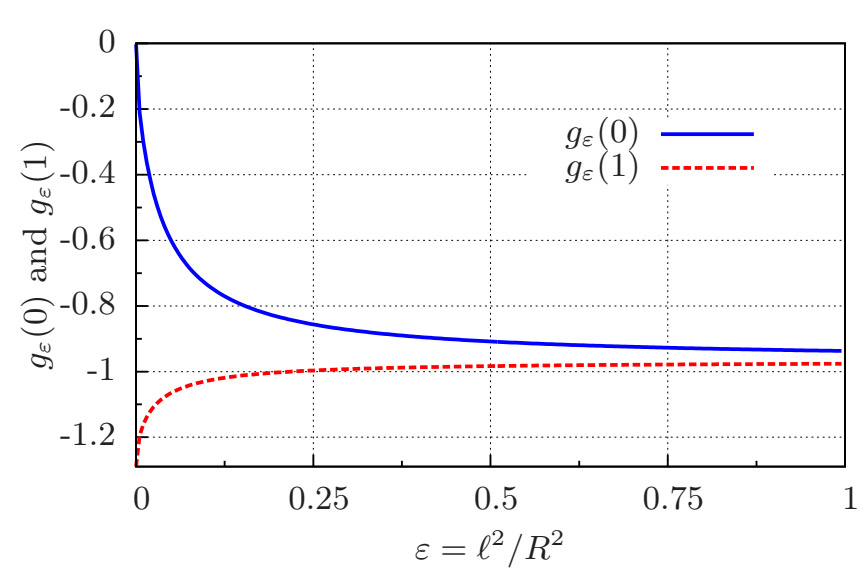

FIG. 7: (Color online.) Reduced rotation angle $g_{\varepsilon} v s$ reduced exchange length $\varepsilon$ : at $r=0$ (solid curve) and $r=1$ (dashed curve).
The rotation angle in the Bloch point is essentially influenced by the exchange parameter $\varepsilon$, see Fig. 7. In the limit case of small particle $(\varepsilon \gg 1)$ the role of exchange is dominant, which results in the constant angle $g_{\infty}=$ $-\sqrt{15} / 4 \approx-0.97$. In the opposite case $\varepsilon \ll 1$, the role of magnetostatic interaction is enhanced and this leads to a nonhomogeneous rotational angle distribution. In the limiting case $g_{0}(0)=0$ and $g_{0}(1)=-\sqrt{5 / 3} \approx-1.3$. Such a limit case is realized in typical soft nanomagnets sized in some tens of nanometers.
* sheka@univ.net.ua

${ }^{1}$ C. Domb and M. S. Green, eds., Phase Transitions and Critical Phenomena, Vol. 7 (Academic Press, 1983) p. 328; K. J. Strandburg, ed., Bond-Orientational Order in Condensed Matter Systems (Springer, 1991) p. 388; F. Yonezawa and T. Ninomiya, Topological Disorder in Condensed Matter, Springer series in solid-state sciences, Vol. 46 (Springer, 1983) p. 253.

2 M. J. Bowick and L. Giomi, "Two-dimensional matter: order, curvature and defects," Advances in Physics 58, 449563 (2009).

3 A. L. Fetter, "Rotating trapped bose-einstein condensates," Rev. Mod. Phys. 81, 647-691 (2009)

4 A. Hubert and R. Schäfer, Magnetic domains: the analysis of magnetic microstructures (Springer-Verlag, Berlin, 1998).

5 J. Stöhr and H. C. Siegmann, Magnetism: From Fundamentals to Nanoscale Dynamics, Springer Series in solidstate sciences, Vol. 152 (Springer-Verlag Berlin Heidelberg, 2006).

${ }^{6}$ A. P. Guimarães, Principles of Nanomagnetism NanoScience and Technology (Springer-Verlag Berlin Heidelberg, 2009).

7 I. I. Naumov, L. Bellaiche, and H. Fu, "Unusual phase transitions in ferroelectric nanodisks and nanorods," $\mathrm{Na}-$ ture 432, 737-740 (2004)

$\therefore$ T. Senthil, A. Vishwanath, L. Balents, S. Sachdev, and M. P. A. Fisher, "Deconfined quantum critical points," Science 303, 1490-1494 (2004), http://www.sciencemag.org/content/303/5663/1490.full.pdf

9 Mermin, "E pluribus boojum: the physicist as neologist," Physics Today 34, 4653 (1981); G. Volovik, The universe in a Helium droplet (Oxford University Press, Oxford, 2003).

10 A. P. Malozemoff and J. C. Slonzewski, Magnetic domain walls in bubble materials (Academic Press, New York, 1979).

11 E. Feldtkeller, "Mikromagnetisch stetige und unstetige magnetisierungskonfigurationen," Zeitschrift für ange- wandte Physik 19, 530-536 (1965)

12 W. Döring, "Point singularities in micromagnetism," J. Appl. Phys. 39, 1006-1007 (1968)

13 Y. P. Kabanov, L. M. Dedukh, and V. I. Nikitenko, "Bloch points in an oscillating bloch line," JETP Lett 49, 637 (1989)

${ }^{14}$ R. Hertel and J. Kirschner, "Magnetic drops in a softmagnetic cylinder," J. Magn. Magn. Mater. 278, L291L297 (2004); F. Porrati and M. Huth, "Micromagnetic structure and vortex core reversal in arrays of iron nanocylinders," Proceedings of the Joint European Magnetic Symposia (JEMS' 04), J. Magn. Magn. Mater. 290-291, 145-148 (2005) H. Niedoba and M. Labrune, "Magnetization reversal via bloch points nucleation in nanowires and dots: a micromagnetic study," The European Physical Journal B - Condensed Matter and Complex Systems V47, 467-478 (2005); L. Vila, M. Darques, A. Encinas, U. Ebels, J.-M. George, G. Faini, A. Thiaville, and L. Piraux, "Magnetic vortices in nanowires with transverse easy axis," Phys. Rev. B 79, 172410 (2009).

15 A. Masseboeuf, T. Jourdan, F. Lancon, P. BayleGuillemaud, and A. Marty, "Probing magnetic singularities during magnetization process in fepd films," Appl. Phys. Lett. 95, 212501 (2009) T. Jourdan, A. Masseboeuf, F. Lançon, P. Bayle-Guillemaud, and A. Marty, "Magnetic bubbles in fepd thin films near saturation," J. Appl. Phys. 106, 073913 (2009).

io A. Thiaville, J. M. Garcia, R. Dittrich, J. Miltat, and T. Schrefl, "Micromagnetic study of bloch-point-mediated vortex core reversal," Phys. Rev. B 67, 094410 (2003).

17 R. Hertel and C. M. Schneider, "Exchange explosions: Magnetization dynamics during vortex-antivortex annihilation," Phys. Rev. Lett. 97, 177202 (2006).

18 X. J. Xing, Y. P. Yu, S. X. Wu, L. M. Xu, and S. W. Li, "Bloch-point-mediated magnetic antivortex core reversal triggered by sudden excitation of a suprathreshold spinpolarized current," Appl. Phys. Lett. 93, 202507 (2008).

19 T. Okuno, K. Shigeto, T. Ono, K. Mibu, and T. Shinjo, "MFM study of magnetic vortex cores in circular permalloy 
dots: behavior in external field," J. Magn. Magn. Mater. 240, 1-6 (2002).

${ }^{20}$ V. Kravchuk and D. Sheka, "Thin ferromagnetic nanodisk in transverse magnetic field," Physics of the Solid State 49, 1923-1931 (2007)

${ }^{21}$ B. Van Waeyenberge, A. Puzic, H. Stoll, K. W. Chou, T. Tyliszczak, R. Hertel, M. Fähnle, H. Bruckl, K. Rott, G. Reiss, I. Neudecker, D. Weiss, C. H. Back, and G. Schütz, "Magnetic vortex core reversal by excitation with short bursts of an alternating field," Nature 444, 461464 (2006).

${ }_{22}$ Q. F. Xiao, J. Rudge, B. C. Choi, Y. K. Hong, and G. Donohoe, "Dynamics of vortex core switching in ferromagnetic nanodisks," Appl. Phys. Lett. 89, 262507 (2006)

${ }^{23}$ R. Hertel, S. Gliga, M. Fähnle, and C. M. Schneider, "Ultrafast nanomagnetic toggle switching of vortex cores," Phys. Rev. Lett. 98, 117201 (2007).

24 K. Yamada, S. Kasai, Y. Nakatani, K. Kobayashi, H. Kohno, A. Thiaville, and T. Ono, "Electrical switching of the vortex core in a magnetic disk," Nat Mater 6, 270-273 (2007)

25 V. P. Kravchuk, D. D. Sheka, Y. Gaididei, and F. G. Mertens, "Controlled vortex core switching in a magnetic nanodisk by a rotating field," J. Appl. Phys. 102, 043908 (2007)

${ }^{20}$ D. D. Sheka, Y. Gaididei, and F. G. Mertens, "Current induced switching of vortex polarity in magnetic nanodisks," Appl. Phys. Lett. 91, 082509 (2007)

27 E. G. Galkina and B. A. Ivanov, "Quantum tunneling in a magnetic vortex in a $2 \mathrm{~d}$ easy-plane magnetic material,"
JETP Lett. 61, 511-514 (1995)

28 Bloch points in magnetic bubbles were classified by Malozemoff and Slonzewski ${ }^{10}$ using the flux $N$ of gyrotropic vector. Simple calculations show that the value of such a flux is opposite to the topological density (7), $N=-Q$.

29 A. Aharoni, Introduction to the theory of Ferromagnetism (Oxford University Press, 1996).

30 R. Elías and A. Verga, "Magnetization structure of a bloch point singularity," The European Physical Journal B Condensed Matter and Complex Systems , 1-8 (2011) 10.1140/epjb/e2011-20146-6.

31 N. Manton and P. Sutcliffe, Topological solitons, Cambridge Monographs on Mathematical Physics (Cambridge University Press, 2004).

32 SLaSi spin-lattice simulations package code available at http://slasi.rpd.univ.kiev.ua

33 We take the integration time step $\Delta t=0.025 \omega_{0}^{-1}$ for the accuracy $\Delta S_{\max }=\max _{i, n}\left|S_{i, n}^{4}-S_{i, n}^{5}\right|<0.01$, where $\omega_{0}=J S^{2} / \hbar, i=x, y, z$ and superscript for $S_{i, n}$ indicates integration order. We used modified RKF45 scheme, where the time step is changed only if accuracy goal for $\Delta S_{\max }$ is not reached for avoiding of noise from step changing.

34 Supplementary video http://slasi.rpd.univ.kiev.ua/ sm/pylypovskyi12.

35 G. Khodenkov, "Exchange reduction of the magnetization modulus in the vicinity of a bloch point," Technical Physics 55, 738-740 (2010), 10.1134/S1063784210050245.

so J. Reinhardt, "Gittertheoretische behandlung von mikromagnetischen singularitseten," Int. J. Magn. 5, 263 (1973)

37 http://cluster.univ.kiev.ua

38 http://icybcluster.org.ua/ 